\section{Psoriasis ist bei Männern meist stärker ausgeprägt}

Hägg D et al. Severity of Psoriasis Differs Between Men and Women: A Study of the Clinical Outcome Measure Psoriasis Area and Severity Index (PASI) in 5438 Swedish Register Patients. Am J Clin Dermatol 2017; 18: 583 - 590

Im Allgemeinen wird Psoriasis als eine Erkrankung angesehen, die keinen Unterschied zwischen den Geschlechtern macht. Andererseits belegen Studien eine oft intensivere Behandlung für männliche Patienten. Und andere Studien zeigen, dass das Geschlecht nicht beeinflusst, ob Patienten eine (intensivere) biologische Behandlung erhalten. Gibt es also doch geschlechtsspezifische Unterschiede in der Ausprägung der Erkrankung?

Eine schwedische Erhebung bestätigt diesen Eindruck. 5438 Patienten, die wegen einer systemischen Behandlung bei mäßiger bis schwerer Psoriasis im schwedischen Register PsoReg registriert wurden, wurden bei ihrer Registrierung mittels Psoriasis Area and Severity Index (PASI) untersucht - ein Index, der die Schwere der Hautveränderungen und ihre Ausdehnung für die Beurteilung kombiniert.

\section{Frauen haben (fast) überall mildere Symptome}

Frauen hatten im Schnitt deutlich niedrigere PASI-Werte als Männer (5,4 vs. 7,3, $\mathrm{p}<0,0001)$ - über alle Altersgruppen hinweg. Das galt für sämtliche Körperregionen außer den Kopf, der bei beiden Geschlechtern ähnlich intensiv betroffen war. Die Autoren vermuten, dass der oft üppigere Haarwuchs bei Frauen und ihr intensiveres Haar- und Hautpflegeregime ihre Kopfhaut mehr gegen die bei Psoriasis günstige Sonneneinstrahlung abschirmt.

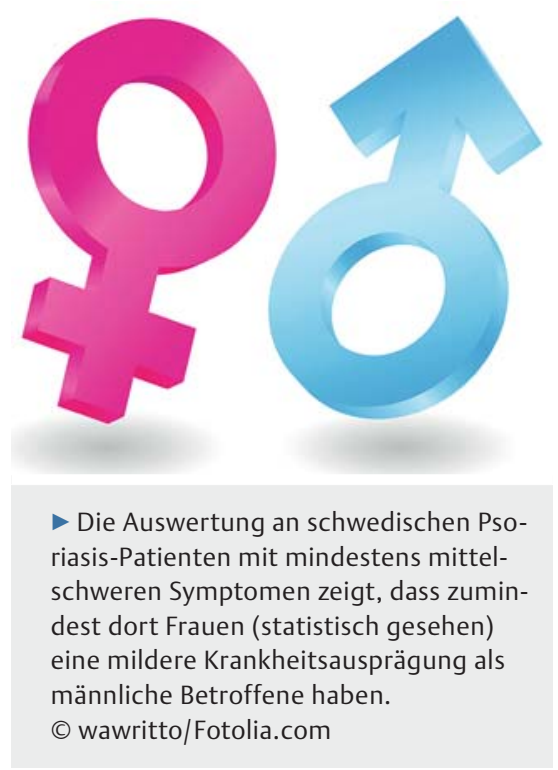

FAZIT

Frauen mit Psoriasis haben (statistisch gesehen) eine mildere Krankheitsausprägung als männliche Betroffene. Das gilt zumindest für schwedische Patienten mit mindestens mittelschweren Symptomen. Weitere Studien müssen zeigen, ob dies auch auf andere Populationen zutrifft. Die ungleiche Geschlechterverteilung in europäischen Psoriasis-Registern spricht aber zumindest dafür, dass nicht nur in Schweden Männer heftiger von Psoriasis betroffen sind als Frauen.

Dr. Nina Drexelius, Hamburg
Dazu passt, dass Männer in den meisten europäischen Registern für systemische Psoriasis-Behandlung in der Überzahl sind: In Schweden und auch in Deutschland machen sie $60 \%$ der registrierten Patienten aus, in mehreren anderen Ländern (darunter Dänemark, Italien, die Niederlande und Spanien) liegt ihr Anteil sogar noch höher (bis 68\%).

Die Ergebnisse von Hägg et al. passen zu Resultaten vorangegangener Studien und erklären u.a., warum Schuppenflechte bei Männern häufiger systemisch behandelt wird. Hierfür wurde erstmals der zuverlässige PASI verwendet, der praktisch nur in randomisierten klinischen Studien Anwendung findet. Andere Daten ließen zur Psoriasis-Geschlechter-Frage nur Vermutungen zu - die jetzt bestätigt wurden.

Zwar wurden nur PsoReg-Patienten in die Studie eingeschlossen - also solche mit einer mindestens mittelschweren Symptomausprägung, die eine systemische Therapie erhalten sollen. Es ist aber davon auszugehen, dass die Erkenntnisse auch für mildere Krankheitsausprägungen gelten. 